\title{
GaAs(2 511$)$ A New Stable Surface within the Stereographic Triangle
}

\author{
L. Geelhaar, J. Márquez, P. Kratzer, and K. Jacobi* \\ Fritz-Haber-Institut der Max-Planck-Gesellschaft, Faradayweg 4-6, D-14195 Berlin, Germany
}

(Received 5 October 2000)

\begin{abstract}
The atomic structure of GaAs(2511), a hitherto unknown stable surface, has been determined by in situ scanning tunneling microscopy and first-principles electronic structure calculations. This orientation is located within the stereographic triangle, i.e., far away from all low-index surfaces. A low-energy $(1 \times 1)$ reconstruction containing arsenic dimers forms on the surface. The analysis of the surface structure shows that, for semiconductor surfaces, the gain in stability due to minimization of the number of dangling bonds is more important than the gain from rendering a semiconducting ground state.
\end{abstract}

DOI: $10.1103 /$ PhysRevLett.86.3815

The chemical environment of atoms at the surface of a crystal is fundamentally different from that of atoms in the bulk, in that some neighboring atoms are missing. Thus, cleavage of a semiconductor crystal results in surface atoms with partially occupied "dangling" bonds that may give rise to metallic states in the bulk band gap. Known surface reconstructions tend to avoid both of these phenomena for energetic reasons, although it should be noted that the famous $\operatorname{Si}(111)-(7 \times 7)$ reconstruction is metallic [1]. It is often believed that only planes with low Miller indices can form low-energy reconstructions, and that a surface of arbitrary orientation decays into facets of these planes. In marked contrast, we report the atomic structure of $\operatorname{GaAs}(2511)$, a hitherto unknown stable surface oriented far away from all low-index surfaces. This finding shines a new light upon the general possibilities that stable surfaces are formed.

The relative orientations of different surfaces are usually visualized in the stereographic triangle with the corners (001), (011), and (111). For bulk-truncated surfaces, planes located on the sides of this triangle are combinations of the planes at the respective corners, and planes within the triangle are composed of all three low-index planes. Hence, the structure of surfaces within the stereographic triangle is expected to be very complex. A few stable high-index semiconductor surfaces are known on the sides of the triangle [2,3], and for $\mathrm{Si}$ and Ge there are reports on stable surfaces within the triangle [4]. However, the structure of the latter was not determined, and results cannot be transferred from elemental to the intrinsically more complex compound semiconductors. To our knowledge, no stable compound semiconductor surface has been observed in this angular region before.

From the abundance of studies on low-index surfaces, guiding principles were extracted that describe the structure of semiconductor surfaces [1], e.g., the electron counting rule (ECR) [5]. The typically more complicated structure of a stable high-index surface offers an ideal testing ground for the general validity of these principles. Thus, further insight can be gained into the structure of surfaces in general. In addition, the structure determination of a stable III-V semiconductor surface within
PACS numbers: 68.35.Bs, 61.50.Ah, 68.37.Ef, 81.05.Ea

the triangle is expected to be very helpful for studies on the technologically promising InAs/GaAs quantum dots [6]. Facets with such orientations have been observed on these structures [7]. Thus, data on related surfaces would yield a better understanding of the shape and formation of quantum dots.

For the present Letter, investigations were carried out on planar GaAs samples whose orientation is located within the stereographic triangle. The experiments were supported by first-principles electronic structure calculations. In a previous study a stable surface was observed in spherical depressions that were ground into GaAs(113)A samples [8]. Because of experimental limitations its structure and Miller indices remained unknown. Here it is demonstrated that the new stable surface has the orientation (2511), and its atomic structure is reported.

Experiments were carried out in a multichamber ultrahigh-vacuum system [9]. Samples with a typical size of $10 \times 10 \mathrm{~mm}^{2}$ were cut from GaAs wafers that are oriented in direction (2511) within $1.5^{\circ}$, cleaned with propanol, and introduced into the UHV system via a loading chamber. After oxide desorption samples were treated with several ion-bombardment and annealing (under $\mathrm{As}_{2}$ flux at $580^{\circ} \mathrm{C}$ ) cycles, which already yielded fairly good diffraction patterns. Homoepitaxial layers 20-200 nm thick were grown by molecular beam epitaxy at a temperature of $580{ }^{\circ} \mathrm{C}$. The $\mathrm{As}_{2}: \mathrm{Ga}$ beam equivalent pressure ratio was 15. After growth, samples were cooled down to $450{ }^{\circ} \mathrm{C}$ and kept at this temperature in the $\mathrm{As}_{2}$ beam for $15 \mathrm{~min}$. Variation of the growth parameters in a wide range yielded always the same reconstruction, only at somewhat different surface qualities. Surfaces were in situ studied by scanning tunneling microscopy (STM) and low-energy electron diffraction (LEED).

Calculations were performed on the basis of densityfunctional theory (DFT) using the local-density approximation in conjunction with pseudopotentials and a plane-wave basis set, as implemented in the computer code FHI98MD [10]. Hamann-type pseudopotentials [11] and a plane-wave cutoff of $136 \mathrm{eV}$ were employed. The surface was modeled by periodic slabs. The slab thickness was $12 \AA$, separated by $12 \AA$ of vacuum. The dangling 

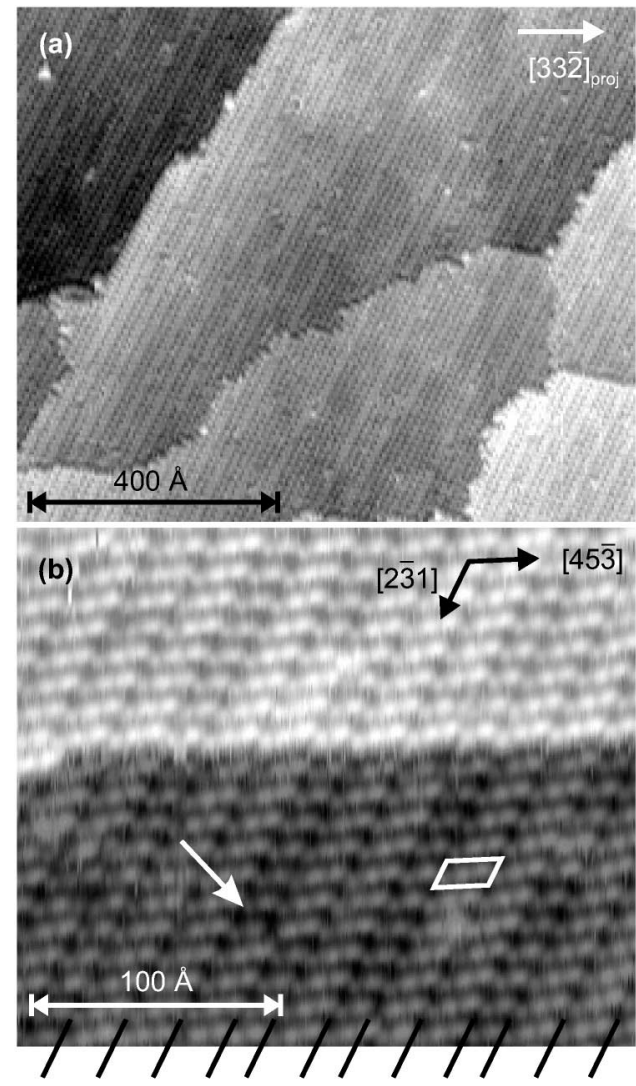

FIG. 1. STM images of GaAs(2511). The projection of the direction [332] , a crystallographic axis in the (113) plane, is indicated for orientation. (a) Large area. $U_{\text {sample }}=-2.5 \mathrm{~V}$, $I=0.1 \mathrm{nA}$. (b) Small area. The unit mesh corresponding to the LEED data is marked by the white parallelogram. The black lines at the bottom indicate the dark lines on the STM image. $U_{\text {sample }}=-2.5 \mathrm{~V}, I=0.3 \mathrm{nA}$.

bonds of As and $\mathrm{Ga}$ at the back side of the slab were passivated with pseudohydrogen atoms of atomic charge $0.75 e$ or $1.25 e$. Surface energies were calculated from the total energies by a subtraction method using reference slabs with hydrogen termination on both sides. By performing test calculations for a thicker slab or a higher cutoff of $200 \mathrm{eV}$, we verified that relative surface energies are converged to within $2 \mathrm{meV} / \AA^{2}$.

A typical overview STM image of the GaAs(2 511) surface is depicted in Fig. 1. The surface consists of terraces that extend over several $100 \AA$. On the terraces there are dark lines running from the lower left to the upper right. A higher resolution [Fig. 1(b)] reveals that these dark lines (cf. markers at bottom image border) separate stripes that are made up from series of either three or two humps. These series are oriented roughly along [45그. The right hand side of the hump series is higher than the left hand side, and hump series on adjacent stripes are shifted in direction [231] with respect to each other. The majority of stripes contains three humps.

LEED images (not shown) reveal an oblique net of sharp spots indicating a high surface quality. The lengths of the real space basis vectors are $(11.1 \pm 0.5) \AA$ and $(21 \pm$ 1) $\AA$, and the enclosed angle is $68^{\circ} \pm 2^{\circ}$. The lengths of the unit mesh marked in Fig. 1(b) are $\sim 11 \AA$ and $\sim 20 \AA$, and the enclosed angle is $\sim 70^{\circ}$. These values are in good agreement with the LEED data. Thus, stripes with only two humps are an element of disorder. Both the LEED and STM results are identical to those from the spherical depression [8], so the same surface is now observed on a planar sample.

On the basis of the STM images a structural model was developed that is presented in Fig. 2. Since the distances between atomic scale features can have only discrete values, the experimental uncertainties were negligible for the construction. This way the Miller indices of the stable surface were determined as (2511). The lengths of the basis vectors are $10.6 \AA$ and $20.0 \AA$, and the enclosed angle is $67.8^{\circ}$. All these values are in excellent agreement with the experimental data.

The unit mesh of the reconstruction contains a series of three As dimers, three As atoms with one dangling bond each, and seven Ga atoms with one dangling bond each. The dimers are seen as humps in the filled state STM images, while the empty Ga dangling bonds are not visible. The electrons from the Ga dangling bonds can be distributed in such a way that all the Ga dangling bonds are emptied and all the As dangling bonds are completely filled. Thus, the proposed structural model fulfills the ECR; i.e., the surface has a semiconducting ground state.

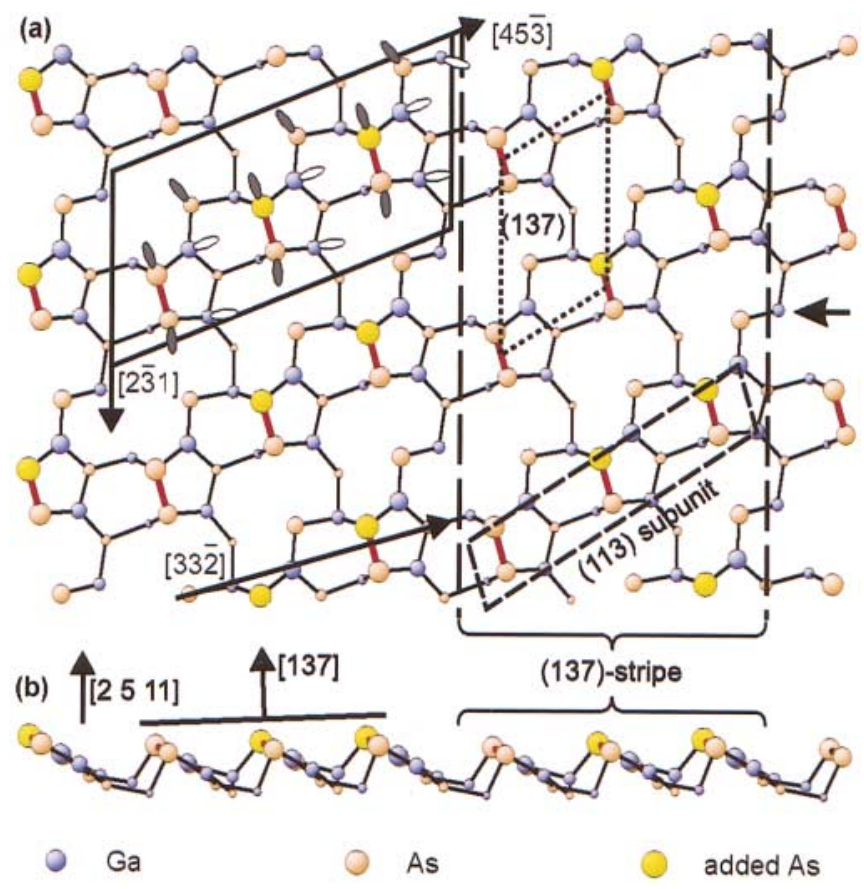

FIG. 2 (color). Structural model of the reconstructed $\operatorname{GaAs}(2511)-(1 \times 1)$ surface. The solid parallelogram indicates the unit mesh. The exact positions of the atoms are the result of the calculations. (a) Top view. (b) Side view. 
The resulting energetic gain is in accord with the observed stability of the GaAs (2511) surface.

In order to construct the model shown in Fig. 2, two As atoms were added to the bulk-truncated surface. This As enrichment is in agreement with the experimental observation that the surface quality was the highest for samples that were annealed after growth in the $\mathrm{As}_{2}$ beam. The number of dangling bonds was reduced by forming dimer bonds between adjacent As atoms. This is in accord with another general principle of surface structure, the saturation and reduction of dangling bonds. The periodicity of the reconstruction remains $(1 \times 1)$, as seen by LEED.

The complicated structure of this reconstruction shall be explained in more detail. The series of three As dimers is inclined with respect to the (2511) plane and forms a (113) subunit [dashed parallelogram in Fig. 2(a)]. However, such an arrangement of three dimers does not exist on the stable $\operatorname{GaAs}(113)$ surface that exhibits an $(8 \times 1)$ reconstruction [3], so this is not a form of faceting into planes of lower surface energy. Because of the geometry of the (2511) plane the dimer bonds are also inclined with respect to this plane. Dimer series that are neighboring in direction [2 $\overline{3} 1]$ form stripes of the orientation (137) [area between vertical dashed lines in Fig. 2(a)]. The dark lines from the lower left to the upper right that are visible on both STM images in Fig. 1 are the trenches between these stripes. There are two Ga atoms with one dangling bond each at every As dimer. The seventh Ga dangling bond is located in the narrow trench between adjacent (137) stripes [arrow on right-hand side of Fig. 2(a)]. Without this Ga dangling bond the ECR would be violated, as it is also the case for a by analogy reconstructed (137) surface with arsenic dimers [dotted unit mesh in Fig. 2(a)]. Thus, the stability of $\operatorname{GaAs}(2511)$ is caused by the balance between (137) stripes of a certain width and one additional Ga dangling bond per unit mesh in the trenches.

To quantify the stability of the reconstructed GaAs(25 11) surface, total energy calculations were performed. For nonstoichiometric surfaces, the surface free energy is a function of the chemical potential of one of the elements, in this case of $\mu_{\mathrm{As}}$, the arsenic chemical potential. The cohesive energy of solid elemental arsenic, $E_{\mathrm{As}}^{\mathrm{coh}}$, constitutes a lower bound for $\mu_{\text {As }}$ (see also Ref. [12]). The surface free energy at zero temperature was determined to $53 \mathrm{meV} / \AA^{2}+0.0107 \AA^{-2} \times\left(E_{\mathrm{As}}^{\mathrm{coh}}-\mu_{\mathrm{As}}\right)$. For As-rich conditions $\left(E_{\mathrm{As}}^{\mathrm{coh}}=\mu_{\mathrm{As}}\right)$, the value for $\operatorname{GaAs}(2511)\left(53 \mathrm{meV} / \AA^{2}\right)$ lies between those for the well-known $c(4 \times 4) \quad\left(45 \mathrm{meV} / \AA^{2}\right)$ and $\beta 2(2 \times 4)$ $\left(55 \mathrm{meV} / \AA^{2}\right)$ reconstructions on $\mathrm{GaAs}(001)$ [12]. The low energy corroborates the suggested structural model for $\operatorname{GaAs}(2511)$.

In order to validate the interpretation of the experimental STM images, they are compared to simulated images obtained on the basis of the calculated geometry. In Fig. 3 the experimental and simulated STM images are presented next to each other. For the simulations, isosurfaces are

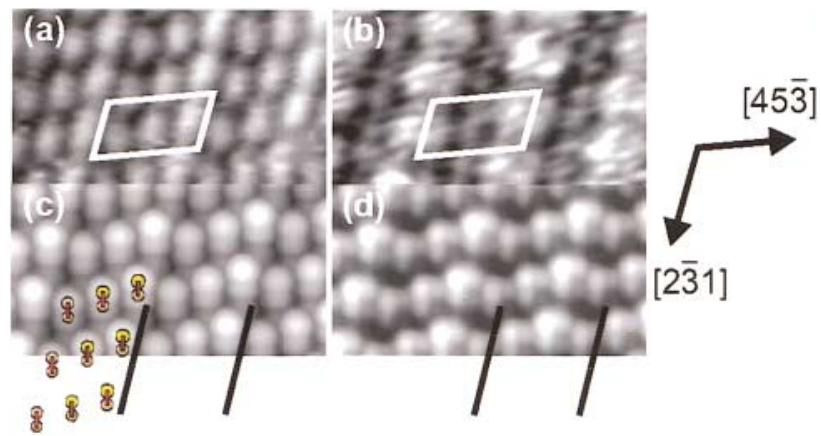

FIG. 3 (color). Experimental and simulated high-resolution STM images of $\operatorname{GaAs}(2511)$. The white parallelograms indicate the unit mesh, and the black lines at the bottom mark the dark lines on the images. (a) Experimental, filled states. $U_{\text {sample }}=-3.0 \mathrm{~V}, I=0.1 \mathrm{nA}$. (b) Experimental, empty states. $U_{\text {sample }}=+2.5 \mathrm{~V}, I=0.3 \mathrm{nA}$. (c) Simulated, filled states. Arsenic dimers of the structural model in overlay. (d) Simulated, empty states.

displayed for a suitably defined local density of states integrated over an energy interval extending from the valence band top to $-1 \mathrm{eV}$ below for the filled state image, and from the bottom of the conduction band to $1 \mathrm{eV}$ above for the empty state image, respectively. This simulation method corresponds to STM images taken in constant current mode [13]. For the comparison it has to be taken into consideration that it is experimentally more difficult to acquire empty state images. The difference between the filled and empty state images lies in which separations between adjacent humps are the most pronounced. In the filled state images, dark lines appear between the highest and lowest humps, as indicated by the markers and discussed before. Thus, three ascending humps form a series. In contrast, in the empty state images there is no distinguishable minimum in intensity between the highest and the lowest humps. Instead, there are dark lines between the lowest and the middle humps, as marked by the black lines at the bottom of Fig. 3(d). Hence, the highest hump forms a group with its two neighboring humps. This feature is visible both in the experimental and in the simulated images. Therefore, for both filled and empty states simulation and experiment are essentially identical, confirming our model.

In the STM images there are also stripes visible that are made up from series of only two As dimers. If such a series of two dimers occurs, it is fairly stable; i.e., the corresponding stripe extends in direction [2 $\overline{3} 1]$ over up to several $100 \AA$ and even across step edges. Hence, these stripes are a large-scale, ordered violation of the surface periodicity. A surface consisting exclusively of two-dimer stripes would have an orientation only slightly different $\left(\leq 1^{\circ}\right)$ from (2511), namely (3715). Thus, on $\operatorname{GaAs}(2511)$ these stripes can be considered either as line defects of the reconstruction or as minimally off-oriented nanofacets. 
In order to understand the occurrence of the two-dimer stripes, computations were also performed for a hypothetical reconstructed GaAs(3715) surface. The unit mesh of this surface looks very similar to the one depicted in Fig. 2, but contains only two As dimers. Its dangling bond density is greater by only $0.1 \%$. However, the GaAs(37 15) surface does not fulfill the ECR. Thus, it is expected to be of higher energy. Since GaAs(2511) and (3715) differ basically only in the fulfillment of the ECR, their simultaneous observation offers a unique test of the relative importance of the guiding principles for semiconductor surfaces. The calculations yielded that the surface energy of GaAs(3715) is $55 \mathrm{meV} / \AA^{2}+$ $0.0097 \mathrm{meV} / \AA^{2} \times\left(E_{\mathrm{As}}^{\mathrm{coh}}-\mu_{\mathrm{As}}\right)$. Surprisingly, the value for As rich conditions $\left(55 \mathrm{meV} / \AA^{2}\right)$ is only insignificantly greater than the respective value for $\operatorname{GaAs}(2511)$. We conclude that the reduction in the number of dangling bonds that is achieved by the common structural motif of arsenic dimers is more important for the stability of a surface than the fulfillment of the ECR. This finding questions the common practice of excluding structural models that violate the ECR in the discussion of semiconductor surface structures. We expect that this observation is generally valid for surfaces of III-V semiconductors.

The calculations show that the thermodynamic driving force for the relaxation of the two-dimer stripes into the regular $(2511)$ structure is small. In addition, the line defect, once it has formed, is stabilized kinetically due to the geometry of the (2511) plane: In order to turn a two-dimer stripe into a three-dimer one, a significant rearrangement of atoms across the whole terrace is necessary. It can indeed be observed in Fig. 1 that a two-dimer stripe changes into a three-dimer stripe (cf. arrow), but to make up for this the neighboring stripe changes from three to two dimers.

Since the two-dimer stripes violate the ECR, they are likely to have metallic surface states. This is supported by the electronic structure calculations for GaAs(3715). Thus, these stripes, embedded in the semiconducting GaAs(2511) surface, could possibly be electronically one-dimensional systems.

In conclusion, this Letter reports the atomic structure of GaAs(2511), the first known stable compound semiconductor surface within the stereographic triangle. The characteristic element of the reconstruction is an inclined series of three As dimers. The surface is perturbed by thin stripes of the nearby orientation (3 715 ). The DFT calculations of the respective surface energies suggest that, for semiconductor surfaces, the gain in stability caused by the minimization of the number of dangling bonds is significantly greater than the one caused by reaching a semiconducting ground state. The discovery of $\operatorname{GaAs}(2511)$ falsifies the old assumption that only surfaces with low Miller indices are stable and raises the question of what other surfaces there may be.

We thank M. Scheffler and G. Ertl for support and P. Geng for technical assistance. This work was supported by the Deutsche Forschungsgemeinschaft (Sonderforschungsbereich 296) and by the German Bundesministerium für Bildung und Forschung under Grant No. 05622 EBA4.

*Corresponding author.

Electronic address: jacobi@fhi-berlin.mpg.de

[1] C. B. Duke, Chem. Rev. 96, 1237 (1996).

[2] J. Da̧browski, H.-J. Müssig, and G. Wolff, Phys. Rev. Lett. 73, 1660 (1994); A. A. Baski, S.C. Erwin, and L. J. Whitman, Science 269, 1556 (1995); S.C. Erwin, A. A. Baski, and L. J. Whitman, Phys. Rev. Lett. 77, 687 (1996); Surf. Sci. 392, 69 (1997); A. Laracuente, S. C. Erwin, and L. J. Whitman, Phys. Rev. Lett. 81, 5177 (1998); J. Márquez et al., ibid. 86, 115 (2001).

[3] M. Wassermeier et al., Phys. Rev. B 51, 14721 (1995); J. Platen et al., J. Appl. Phys. 85, 3597 (1999).

[4] T. T. Tsong, D. L. Feng, and H. M. Liu, Surf. Sci. 199, 421 (1988); Z. Gai et al., Phys. Rev. B 56, 12308 (1997); 57, R6795 (1998); 57, R15 060 (1998); 59, 15230 (1999).

[5] W. A. Harrison, J. Vac. Sci. Technol. 16, 1492 (1979); M. D. Pashley, Phys. Rev. B 40, 10481 (1989).

[6] D. Bimberg, M. Grundmann, and N. N. Ledentsov, Quantum Dot Heterostructures (Wiley, Chichester, 1999).

[7] H. Lee et al., Appl. Phys. Lett. 72, 812 (1998); Y. Hasegawa et al., ibid. 72, 2265 (1998); J. Márquez, L. Geelhaar, and K. Jacobi, Appl. Phys. Lett. (to be published).

[8] L. Geelhaar, J. Márquez, and K. Jacobi, Phys. Rev. B 62, 6908 (2000).

[9] P. Geng et al., Rev. Sci. Instrum. 71, 504 (2000).

[10] M. Bockstedte et al., Comput. Phys. Commun. 107, 187 (1997).

[11] M. Fuchs and M. Scheffler, Comput. Phys. Commun. 116, 67 (1999)

[12] N. Moll et al., Phys. Rev. B 54, 8844 (1996).

[13] J. Tersoff and D. R. Hamann, Phys. Rev. B 31, 805 (1985). 